\title{
PRINCIPLES AND APPLICATIONS OF SUPERCONDUCTING QUANTUM INTERFERENCE DEVICES
}


This page is intentionally left blank 


\title{
PRINCIPLES AND APPLICATIONS OF SUPERCONDUCTING QUANTUM INTERFERENCE DEVICES
}

\author{
Edited by Antonio Barone \\ DIPARTIMENTO SCIENZE FISICHE \\ UNIVERSITÀ DI NAPOLI "FREDERICO II" \\ NAPLES, ITALY \\ C.N.R. NATIONAL PROJECT \\ SUPERCONDUCTIVITY AND CRYOGENIC \\ TECHNOLOGIES
}




\section{Published by}

World Scientific Publishing Co. Pte. Ltd.

P O Box 128, Farrer Road, Singapore 9128

USA office: Suite 1B, 1060 Main Street, River Edge, NJ 07661

UK office: 73 Lynton Mead, Totteridge, London N20 8DH

\section{PRINCIPLES AND APPLICATIONS OF SUPERCONDUCTING QUANTUM INTERFERENCE DEVICES}

Copyright $\odot 1992$ by World Scientific Publishing Co. Pte. Led.

All rights reserved. This book, or parts thereof, may not be reproduced in any form or by any means, electronic or mechanical including photocopying, recording or any information storage and retrieval system now known or to be invented, without written permission from the Publisher.

ISBN 981-02-0911-8

Printed in Singapore by Utopia Press. 
When I was invited to act as editor of a book concerning SQUIDs, I considered the idea very stimulating as well as extremely challenging. Indeed, to what extent it was possible to make an interesting and comprehensive volume on the subject was a nontrivial question.

As a result, I believe that the contribution of excellent authors, together with the selection of the topics, guarantees for the high quality standard of the present book. However, it certainly cannot be considered exhaustive or conclusive, this matter continuously growing.

The volume lies halfway between a textbook and a multi-author collection of critical reviews. The latter aspect emerges in the introductory material of each chapter which can somewhat overlap. However, without preventing a large scale coherence of the whole text, this leads to rather self contained chapters. This choice can better provide "individual" messages for the various scientific communities interested in the physics of SQUIDs and their specific applications. Indeed the smooth introduction of each chapter allows the reader to skip, at least in a first reading, the other ones without a serious lack of continuity.

The first chapter provides an extensive background for the comprehension of the physics underlying the SQUID structures focusing mainly on the D.C. configuration and leaving classical and modern aspects of the R.F. devices to the second chapter. The description of very advanced technology and important aspects of device fabrication are given in chapter 3 . In chapter 4 , an extensive discussion on the most important topic of Biomagnetism, the Neuromagnetic studies, is given in detail. Chapter 5 deals with a further new challenging area, namely the SQUID technology for space applications. A description of the variety of applications of SQUIDs to the world of fundamental physics is contained in chapter 6 . The last chapter gives an account of the status of high $T_{c}$ Superconducting SQUIDs, which includes extremely interesting results and important notions for projections of this extremely stimulating topic.

As it will appear from the reading of the book, SQUID's technology has reached a degree of maturity which legitimates the great interest and research efforts devoted to the topic during the last two decades. This not only for the stimulating underlying physics but also for the concrete perspectives of industrial applications to which high- $T_{\mathrm{c}}$ materials add a further interest. Indeed, as stated by a great pioneer of the field, John Clarke, "given the world-wide effort on the new superconductors there is every reason to be optimistic about the long-term future of SQUIDs based on these materials". 
I hope that the present book, providing both basic aspects and recent progresses of SQUIDs technology, might of fer a realistic and stimulating picture of the state-of-theart, contributing towards a further development of the field toward a variety of applications.

I gladly take the opportunity to thank, here, the several authors who have contributed to this volume and the directors of the various museums and organizations who kindly provided the photographs and slides used for the chapters separations. Thanks are also due to Mrs. Anna Maria Mazzarella for her valuable assistance throughout the editing.

*Professor of Structure of Matter, Physical Sciences Department, Faculty of Engineering, University of Naples and Director of the Project "Superconductive and Cryogenic Technologies" of the Italian National Research Council. 


\section{CONTRIBUTORS TO THE VOLUME}

B. Cabrera, Physics Department, Stanford University, Stanford, California, USA

P. Carelli, Dipartimento di Energetica, Universita di L'Aquila, L'Aquila and I.E.S.S., CNR, Rome, Italy

P. Chaudhari, IBM Research Division, T. J. Watson Research Center, Yorktown Heights, New York, N.Y., USA

R. Gross, Lehrstuhl Experimentalphysik II, Universitat Tübingen, Tübingen, Germany

M. Koyanagi, Electrotechnical Laboratory, Umezono, Tsukuba, Ibaraki, Japan

L. Narici, Dipartimento di Fisica, Università di Roma "Tor Vergata", Rome, Italy

M. Nisenoff, Electronics Science and Technology Division, Naval Research Laboratory, Washington, D.C., USA

G. Paterno, ENEA, C.R.E. Frascati, Rome, Italy

G. L. Romani, Istituto di Fisica Medica, Università "G. D'Annunzio", Chieti, Italy and I.E.S.S., CNR, Rome, Italy

V. I. Shnyrkov, Institute for Low Temperature Physics and Engineering, Ukr. SSR Academy of Sciences, Kharkov

S. Takada, Electrotechnical Laboratory, Umezono, Tsukuba, Ibaraki, Japan

G. M. Tsoi, Institute for Low Temperature Physics and Engineering, Ukr. SSR Academy of Sciences, Kharkov 
This page is intentionally left blank 
Preface $\quad v$

Contributors to the volume vii

1. Macroscopic Quantum Interference: DC-SQUID 3

P. CARELLI and G. PATERNO

1. Introduction 4

2. Flux Quantization 5

3. Josephson Effect 9

4. Superconducting Interferometers $\quad 22$

5. DC-SQUID: Operation 44

$\begin{array}{ll}\text { References } & 67\end{array}$

2. Signal and Noise Characteristics of RF-SQUIDs 77

V. I. SHNYRKOV and G. M. TSOI

1. Introduction $\quad 78$

2. RF-SQUIDs 93

3. SQUIDs made of High-Temperature Superconductors (HTSC) 125 References 143

3. Fabrication of Integrated DC-SQUID with Refractory Tunnel Junctions

S. TAKADA and M. KOYANAGI

1. Introduction $\quad 152$

2. Principle of the DC-SQUID 153

3. Design of DC-SQUID Integrated with Refractory Josephson Junction Process

4. Fabrication of the DC-SQUID 164

5. Operation of the Fabricated DC-SQUID 174

6. DC-SQUID with $\mathrm{NbN} / \mathrm{MgO} / \mathrm{NbN}$ Tunnel Junctions 178

7. Application to Measure a Magnetic Field of Human Body Activity 180 References 
4. SQUIDs in the Study of Cerebral Magnetic Field 189 G. L. ROMANI and L. NARICI

1. Introduction

2. Historical Overview

3. Neuromagnetic Fields and Ambient Noise

4. Detectors

5. Detection Coils

6. Modeling

7. Spatial Configuration of the Sensors

8. Source Localization

9. Signal Processing

10. Data Analysis

11. Examples of Neuromagnetic Studies

12. Conclusions

References

5. Space Applications of Superconductivity M. NISENOFF

1. Introduction

2. Fundamentals of Superconductivity

3. High Temperature Superconductivity

4. Cryogenic Refrigeration

5. Magnets and Solenoids

6. High Frequency Applications in Space

7. Instrumentation

8. Analog and Digital Processing $\quad 319$

9. Nuclear Particle Detection 327

10. High Temperature Superconductivity Space Experiment 331

11. Conclusions

6. Fundamental Physics Experiments Using SQUIDs B. CABRERA

1. Introduction

2. Ginzburg-Landau Theory and Flux Quantization

3. Ultra-low Magnetic Fields 
4. Absolute Flux Quantization Experiment 359

5. Measuring the Cooper Pair Mass 365

6. Search for Magnetic Monopoles 382

7. Reducing 1/f Noise in SQUID Measurements 405

8. Closing Thoughts 413 References $\quad 414$

7. SQUIDs Employing High Temperature Superconductors 419 R. GROSS and P. CHAUDARI

1. Introduction $\quad 420$

2. Impact of High Temperature Superconductivity on SQUIDs 421

3. Key Elements on Thin Film dc-SQUIDs-Present Status 424

4. Design of dc-SQUIDs with Single Grain Boundary Junctions 450

5. Transport Characteristics of Grain Boundary Junction dc-SQUIDs 452

6. Noise Properties of High- $T_{C}$ SQUIDs 459 References $\quad 472$ 